\title{
Chromatin regulates expression of small RNAs to help maintain transposon methylome homeostasis in Arabidopsis
}

Ranjith K. Papareddy, Katalin Páldi ${ }^{\dagger}$, Subramanian Paulraj ${ }^{\dagger}$, Ping Kao, Stefan Lutzmayer and Michael D. Nodine* (D)

\author{
* Correspondence: michael.nodine@ \\ gmi.oeaw.ac.at \\ ${ }^{\dagger}$ Katalin Páldi and Subramanian \\ Paulraj contributed equally to this \\ work. \\ Gregor Mendel Institute (GMI), \\ Austrian Academy of Sciences, \\ Vienna Biocenter (VBC), Dr. \\ Bohr-Gasse 3, 1030 Vienna, Austria
}

\begin{abstract}
Background: Eukaryotic genomes are partitioned into euchromatic and heterochromatic domains to regulate gene expression and other fundamental cellular processes. However, chromatin is dynamic during growth and development and must be properly re-established after its decondensation. Small interfering RNAs (siRNAs) promote heterochromatin formation, but little is known about how chromatin regulates siRNA expression.

Results: We demonstrate that thousands of transposable elements (TES) produce exceptionally high levels of siRNAs in Arabidopsis thaliana embryos. TEs generate siRNAs throughout embryogenesis according to two distinct patterns depending on whether they are located in euchromatic or heterochromatic regions of the genome. siRNA precursors are transcribed in embryos, and siRNAs are required to direct the re-establishment of DNA methylation on TEs from which they are derived in the new generation. Decondensed chromatin also permits the production of 24-nt siRNAs from heterochromatic TEs during post-embryogenesis, and siRNA production from bipartite-classified TEs is controlled by their chromatin states.

Conclusions: Decondensation of heterochromatin in response to developmental, and perhaps environmental, cues promotes the transcription and function of siRNAs in plants. Our results indicate that chromatin-mediated siRNA transcription provides a cell-autonomous homeostatic control mechanism to help reconstitute pre-existing chromatin states during growth and development including those that ensure silencing of TEs in the future germ line.
\end{abstract}

Keywords: Small RNAs, DNA methylation, Chromatin, Epigenetics, Linker histone $\mathrm{H} 1$, Plant embryogenesis, RNAi, Transposable elements

\section{Background}

Eukaryotic genomes are partitioned into euchromatic and heterochromatic domains [1, 2]. Euchromatic regions are enriched for genes and provide a transcriptionally permissive state. Heterochromatic regions are densely packed, or condensed, regions of the genome that are typically transcriptionally quiescent and characterized by highly

(c) The Author(s). 2020 Open Access This article is licensed under a Creative Commons Attribution 4.0 International License, which permits use, sharing, adaptation, distribution and reproduction in any medium or format, as long as you give appropriate credit to the original author(s) and the source, provide a link to the Creative Commons licence, and indicate if changes were made. The images or other third party material in this article are included in the article's Creative Commons licence, unless indicated otherwise in a credit line to the material. If material is not included in the article's Creative Commons licence and your intended use is not permitted by statutory regulation or exceeds the permitted use, you will need to obtain permission directly from the copyright holder. To view a copy of this licence, visit http://creativecommons.org/licenses/by/4.0/. The Creative Commons Public Domain Dedication waiver (http://creativecommons.org/publicdomain/zero/1.0/) applies to the data made available in this article, unless otherwise stated in a credit line to the data. 
repetitive DNA and transposable elements [3, 4]. Heterochromatin formation is promoted by various pathways including those affecting covalent modifications of histones, which package DNA into nucleosomes, as well as cytosine methylation [5-7]. Chromatin states are re-established after fertilization of egg and sperm in diverse animals [8-12], and histone reprogramming has also been observed in plants shortly after fertilization [13, 14]. However, little is known about how heterochromatin-promoting pathways respond to labile chromatin states shortly after fertilization to help reestablish euchromatic and heterochromatic states.

Small RNA-based pathways promote heterochromatin formation and associated transcriptional silencing in animals, fungi, and plants [15]. Plants employ 24-nucleotide (nt) small interfering RNAs (siRNAs) to help promote silencing of repetitive elements including TEs, and thus prevent DNA mutations caused by TE mobilization. During canonical RNA-directed DNA methylation (RdDM), RNA Polymerase IV (Pol IV) is recruited to target loci and generates transcripts that are co-transcriptionally converted into double-stranded RNAs by RNA-DEPENDENT RNA POLYMERASE 2 (RDR2) [16-21]. The resulting double-stranded RNAs are then processed into 23-nt/24-nt RNA duplexes by the DICER-LIKE 3 (DCL3) endoribonuclease [22]. The 24-nt strand of the duplex binds to ARGONAUTE 4 (AGO4) and guides AGO4 and associated proteins to siRNA-complementary sites contained within noncoding RNAs produced by RNA Polymerase V [23, 24]. DOMAINS REARRANGED METHYLTRANSFERASES 1/ 2 (DRM1/2) is then recruited to target loci, which results in the de novo methylation of cytosines in the CG, CHG, and CHH contexts (where $\mathrm{H} \neq \mathrm{G}$ ) $[25,26]$. Because CG and CHG methylation are maintained independently of siRNAs by DNA METHYLTRANSF ERASE 1 (MET1) and CHROMOMETHYLASE 3 (CMT3), respectively, CHH methylation is a good indicator of RdDM activities [20]. Nevertheless, CHH methylation can also be maintained independently of siRNAs by CMT2 in post-embryonic tissues, and this occurs mostly on the bodies of long TEs that are densely packed in nucleosomes and inaccessible to DRM2 [27].

Although sRNAs promote heterochromatin formation in diverse eukaryotic species, little is known about how chromatin states regulate small RNA production in animals and plants. Plant gametes and associated companion cells that support the gametes are contained within multicellular haploid gametophytes [28]. Current models propose that the large-scale chromatin decondensation observed in terminally differentiated companion cells facilitates TE transcription, and the resulting transcripts serve as substrates for RDR1/6 and DCLs to generate 20-22-nt siRNAs that move into gametes and stabilize TE silencing [29-33]. The endosperm and embryo are products of double-fertilization, and it has also been suggested that hypomethylation of endosperm promotes the production of siRNAs, which then move into the embryo to mediate TE methylation [31,34]. $\mathrm{RdDM}$ is required for the progressive methylation of a few target loci during Arabidopsis thaliana (Arabidopsis) embryogenesis [35], and late-staged embryos are $\mathrm{CHH}$ hyper-methylated compared to other tissues [36-38]. However, the dynamics of embryonic RdDM have not been reported genome-wide in plants due to the difficulty in generating genome-wide profiles of siRNAs and methylomes from early embryos, which are small and deeply embedded within maternal seed tissues. More generally, it is virtually unknown how chromatin states may promote the de novo production of 
sRNAs to help re-establish TE silencing cell-autonomously including in the future germ-line.

\section{Results}

\section{Embryos are enriched for transposon-derived small RNAs}

We recently developed a low-input small RNA sequencing (sRNA-seq) method to profile small RNA dynamics during eight stages of Arabidopsis embryogenesis, as well as floral buds and leaves [39] (Fig. 1a and Additional file 1: Table S1). We previously

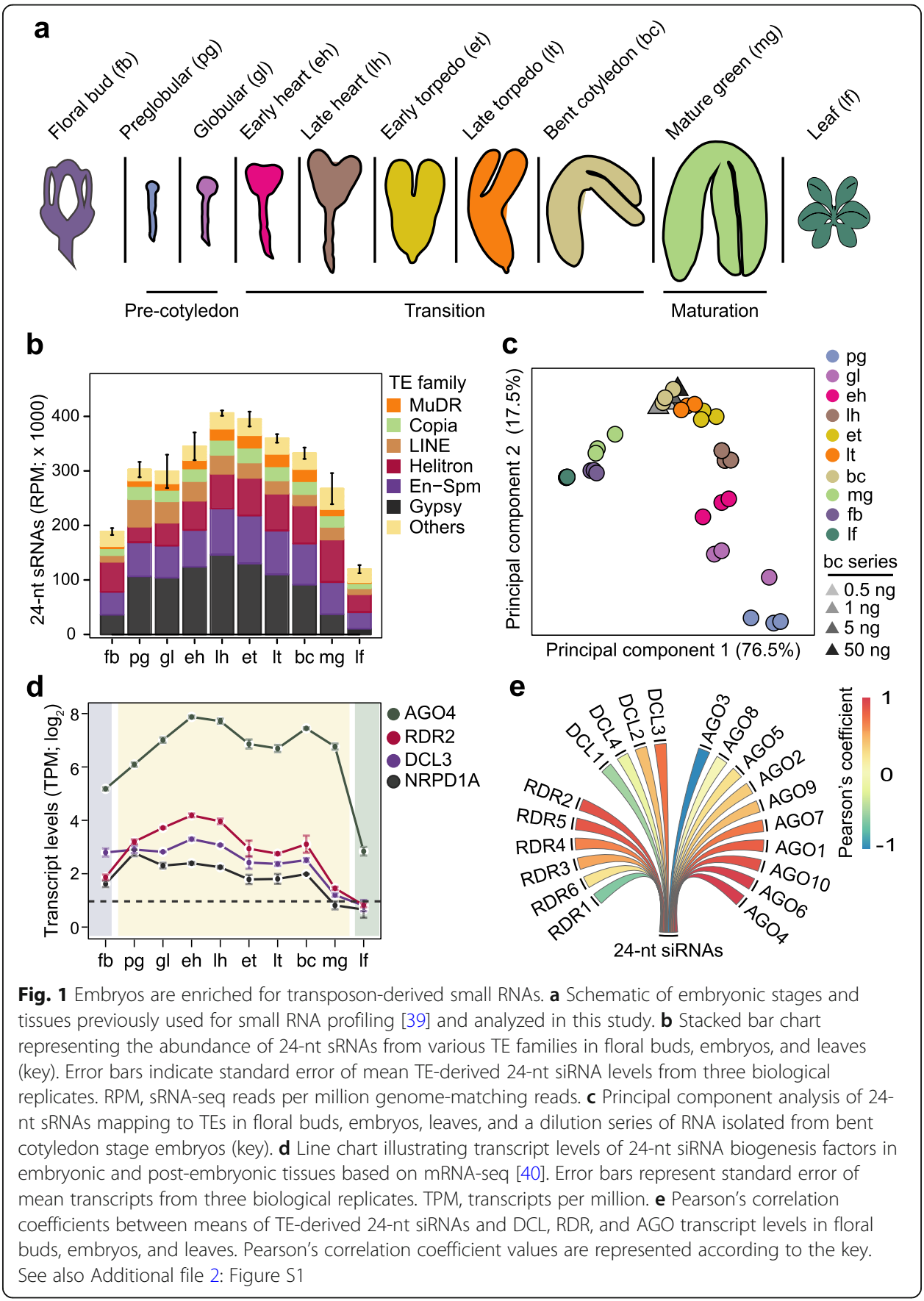


focused on the $\sim 21$-nt microRNA class of small RNAs involved in post-transcriptional regulation [39], but noticed that the vast majority of TE-derived small RNAs were 24nt long and highly enriched in embryos compared to floral bud or leaf tissues (Fig. 1b and Additional file 2: Figure S1A). Small RNAs were detected from 28,087 TEs and the highest amounts from several families including Gypsy, MuDR, and En-Spm were detected during mid-embryogenesis. The levels of TE-derived 24-nt sRNAs were highly correlated among biological replicates from floral bud, embryonic, and leaf tissues indicating stage- and tissue-specific sRNA populations (Fig. 1c and Additional file 2: Figure S1B). Moreover, principal component analysis revealed that $76.5 \%$ and $17.5 \%$ of the variation in TE-derived 24-nt siRNAs was accounted for by principal components 1 and 2, respectively. Principal component 1 distinctly separated post-embryonic and mature green embryo stages from pre-maturation embryonic stages, whereas principal component 2 stratified the pre-maturation embryonic samples according to developmental stage. Libraries prepared from 50, 5, 1, or $0.5 \mathrm{ng}$ of RNA isolated from bent cotyledon embryos clustered together with the biological replicates generated from $\geq$ $500 \mathrm{ng}$ of bent cotyledon RNA (Fig. 1c and Additional file 2: Figure S1B). This indicated that the vast majority of variation observed in 24-nt embryonic and post-embryonic siRNA populations was biological rather than technical.

Small interfering RNAs involved in RdDM are typically 24-nt long and begin with a 5 ' adenine [41]. Accordingly, adenosines were the dominant first base of 24-nt sRNAs in embryos (Additional file 2: Figure S1C), and the levels of embryonic 24-nt sRNAs were most highly correlated with the levels of transcripts encoding key canonical RdDM components such as RDR2, DCL3, and AGO4, which were also enriched in developing embryos (Fig. 1d, e) [20, 40]. Therefore, canonical 24-nt siRNAs are highly enriched in embryos, exhibit distinct developmental dynamics, and upon maturation become similar to post-embryonic siRNA populations.

\section{Small RNAs from euchromatic and heterochromatic transposons exhibit distinct developmental dynamics}

To examine the temporal dynamics of embryonic siRNAs mapping to TEs in more detail, we used mclust [42] to define eight unique clusters of the 31,189 TAIR10annotated TEs based on their 24-nt siRNA levels (Additional file 2: Figure S2A, B). At least 2 sRNA-seq reads per million genome-matching reads were detected for 11,845 TEs, and these were grouped into either class A (6116 TEs; $19.6 \%$ of total) or class B (5729 TEs, 18.4\% of total) based on the dynamics of their corresponding siRNAs between floral buds, developing embryos, and leaves (Fig. 2a, b, Additional file 3: Table S3, and Additional file 2: Figure S2B). The remaining 19,344 TEs $(62.0 \%$ of total) were considered siRNA-depleted (Fig. 2a and Additional file 2: Figure S2B, C) and served as negative controls. Small interfering RNAs from class A TEs had low levels in preglobular embryos that were increased at the globular stage, and were stable until sharply increasing at the mature green stage and then remained at high levels in leaves and floral buds (Fig. 2a-c). In contrast, class B TEs produced large amounts of siRNAs already in preglobular embryos, then continued to gradually increase through mid-embryogenesis and were strongly reduced in mature embryos, leaves, and floral buds (Fig. 2a-c). 

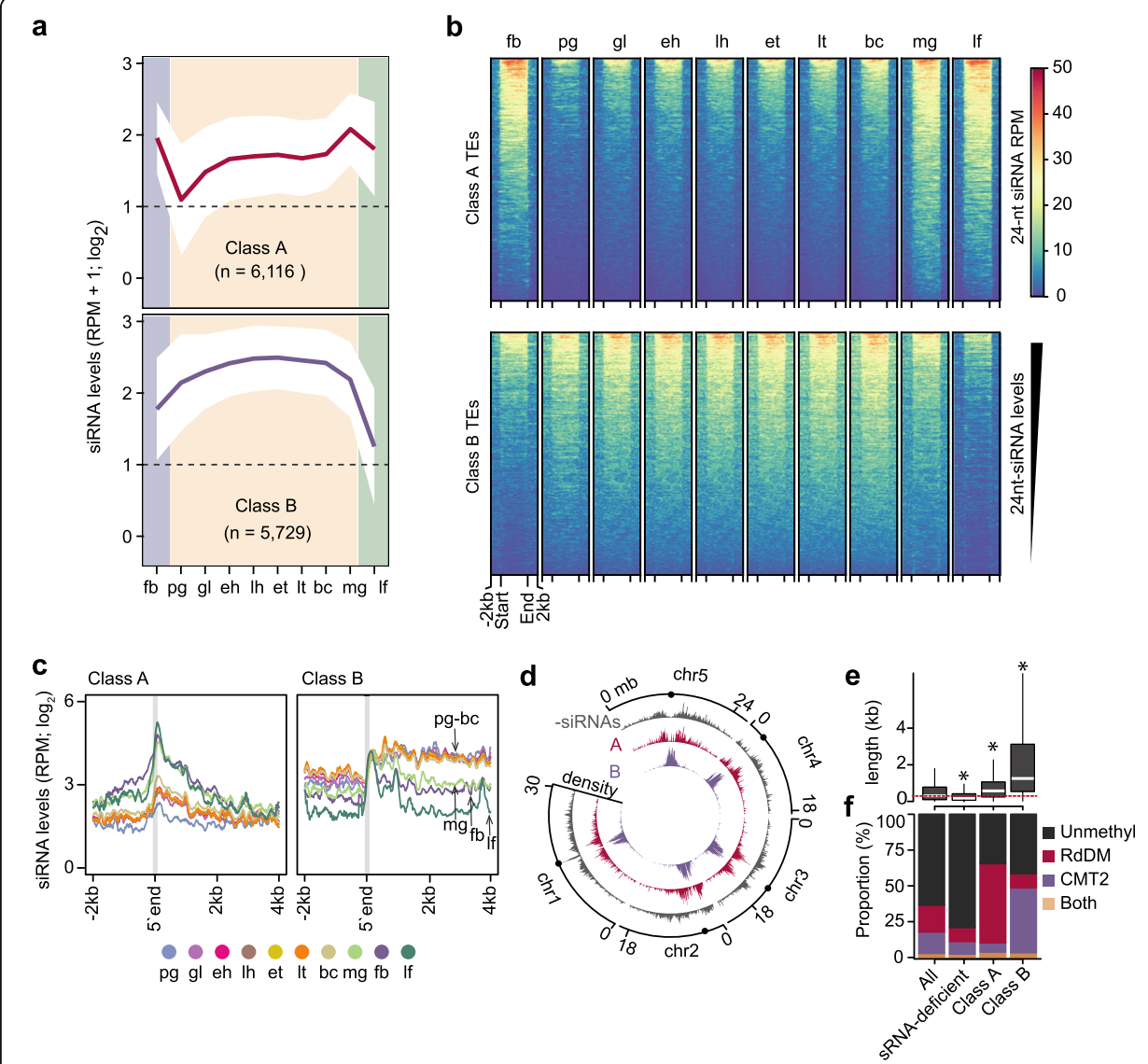

Fig. 2 Small RNAs from euchromatic and heterochromatic transposons exhibit distinct developmental dynamics. a Line graphs illustrating 24-nt siRNA levels from class A and B TEs in embryonic and postembryonic tissues. Dashed lines represent the detection criteria used to select TEs yielding siRNAs (2 RPM, reads per million genome-matching reads). The number of TEs belonging to each class are indicated. Polygons represent the standard deviation of mean 24-nt siRNA levels. fb, floral buds; pg, preglobular; gl, globular; eh, early heart; Ih, late heart; et, early torpedo; It, late torpedo; bc, bent cotyledon; mg, mature green; If, leaf. b Heat map depicting 24-nt siRNA levels (RPM) from class A and B TEs across development. siRNA levels from $\pm 2 \mathrm{~kb}$ of TEs are color-coded according to the key, and samples are labeled as in $\mathbf{a}$. Rows were ordered based on total 24-nt siRNA levels. c Line metaplot showing 24-nt siRNA levels from class A and B TEs. TEs were aligned on their $5^{\prime}$ ends, which are indicated by the gray vertical line. Samples are labeled according to the key and as in a. d Circos plot representing densities of siRNA-deficient (-siRNAs; gray), class A (red) and class B (purple) TEs across the five Arabidopsis chromosomes (chr). Centromeres are indicated by dots. e Boxplot of TE lengths for either all annotated, siRNA-deficient, class A, or class B TEs. Dashed red line represents the median size of all annotated TEs. Thick horizontal bars indicate medians, and the top and bottom edges of the box indicate the 75th and 25th percentiles, respectively. kb, kilobases; $P$ values $<0.0001$ based on Mann-Whitney $U$ test of differences between all annotated and TE classes are represented by *. $\mathbf{f}$ Stacked bar charts illustrating proportion of TEs that are CHH hypomethylated in drm 1/ drm2 (RdDM; red), cmt2 (CMT2; purple), both drm1/drm2 and cmt2 (both; yellow) or which were not methylated (unmethyl; black) in leaves [26]. See also Additional file 2: Figure S2

Class A and B TEs have distinct features. Class A TEs are short and dispersed along pericentromeric and euchromatic regions of chromosomes (Fig. 2d, e and Additional file 2: Figure S2D). In contrast, class B TEs are generally longer, concentrated in heterochromatic centromeres, and especially in embryos, siRNAs are generated from throughout whole TEs (Fig. 2c-e and Additional file 2: Figure S2D). Transposons targeted by DRM2 and CMT2 are distinct to euchromatic and heterochromatic domains of the genome, respectively [27]. The sizes and genomic locations of class A and B TEs are 
characteristic of TEs respectively methylated by either the RdDM or CMT2 pathways in post-embryonic tissues [26, 27, 43]. Indeed, class A TEs were enriched for TEs with reduced methylation in RdDM-defective drm1/2 mutant leaves including HAT, SINE, SADHU, and other short TE families, whereas class B TEs were enriched for TEs with reduced methylation in $c m t 2$ mutant leaves including MuDR, En-Spm, and long terminal repeat families such as Gypsy and Copia (Fig. 2f and Additional file 2: Figure S2E, F). In addition, class B TEs have heterochromatic features such as high levels of GC content, nucleosome occupancy, HISTONE 3 LYSINE 9 di-methylation (H3K9me2), and linker histone 1 (H1) compared to class A TEs (Additional file 2: Figure S2G). Altogether, we identified two distinct classes of TEs based on the levels of siRNAs they produce during development: euchromatic TEs (i.e., class A) that progressively generate siRNAs during embryogenesis and are methylated by the siRNAdependent $\mathrm{RdDM}$ pathway in post-embryonic tissues, and heterochromatic TEs (i.e., class B) that produce very large amounts of siRNAs during embryogenesis prior to maturation and are methylated independently of siRNAs in post-embryonic tissues.

\section{Embryonic methylome dynamics}

During RdDM, 24-nt siRNAs are loaded onto ARGONAUTE proteins and serve as sequence-specific guides for the recruitment of methyltransferases to target loci. siRNA-directed methylation of TEs contributes to their transcriptional silencing and immobilization and limits their mutagenic potential [44-47]. To investigate the functions of embryonic 24-nt siRNAs, we adapted a whole-genome bisulfite sequencing approach called methylC-seq [48] to profile methylomes at single-base resolution from the low amounts of DNA available from early Arabidopsis embryos (see the "Methods" section) (Additional file 1: Table S1). Comparisons of methylomes generated with 0.1, 0.5 , 1 , or $\sim 4 \mathrm{ng}$ of genomic DNA isolated from bent cotyledon embryos had nearly identical cytosine methylation levels indicating that there was low variability of this method when using different amounts of input DNA (Additional file 2: Figure S3A). Therefore, we used this robust low-input methylC-seq method to profile methylomes from 8-cell/16-cell (preglobular; 3 days after pollination [DAP]), early heart (4 DAP) and bent-cotyledon (8 DAP) embryos, as well as leaves and floral buds. We compared these datasets with publicly available methylomes generated from sperm [32], or latestaged embryos from early torpedo [49], mid-torpedo to early maturation [31], or mature green [37] stages. Because methylation of cytosines in the $\mathrm{CHH}$ context $(\mathrm{mCHH}$, where $\mathrm{H} \neq \mathrm{G}$ ) is a hallmark of siRNA-directed DNA methylation [20], we focused on $\mathrm{CHH}$ methylation. More specifically, the Arabidopsis genome was divided into 50-kb bins, and the mean-weighted $\mathrm{CHH}$ methylation rates from reproductive, embryonic, and vegetative tissues were calculated for each bin (Additional file 2: Figure S3B). Consistent with previous studies, $\mathrm{CHH}$ methylation gradually increased during embryogenesis until peaking at the maturation stage and then decreased in leaves (Additional file 2: Figure S3B) [35, 37, 38]. As expected, $\mathrm{CHH}$ methylation was most prominent at pericentromeric and centromeric regions densely populated with euchromatic and heterochromatic TEs that were enriched for siRNAs during embryogenesis (Additional file 2: Figure S3B). Relative to sperm, euchromatic TEs had low $\mathrm{CHH}$ methylation levels in early embryos that increased during embryogenesis and were hypermethylated relative 
to leaves in 8 DAP bent-cotyledon-staged embryos (Fig. 3a). In contrast, $\mathrm{CHH}$ methylation was barely detectable from heterochromatic TEs in sperm, but then increased in early embryos and became hypermethylated relative to leaves by 6 DAP in early torpedo-staged embryos (Fig. 3b). Therefore, $\mathrm{CHH}$ methylation is established on both euchromatic and heterochromatic TEs during early embryogenesis, and TEs become hypermethylated relative to post-embryonic tissues at late stages of embryogenesis.

To examine embryonic methylation dynamics in more detail, we identified significant differentially methylated regions (DMRs) by pairwise comparisons between six embryonic stages (preglobular, early heart, early torpedo, bent cotyledon, late torpedo-toearly mature green and mature green) (see the "Methods" section). We found 21,361 embryonic CHH DMRs with a median size of approximately 100-bp (Additional file 4:

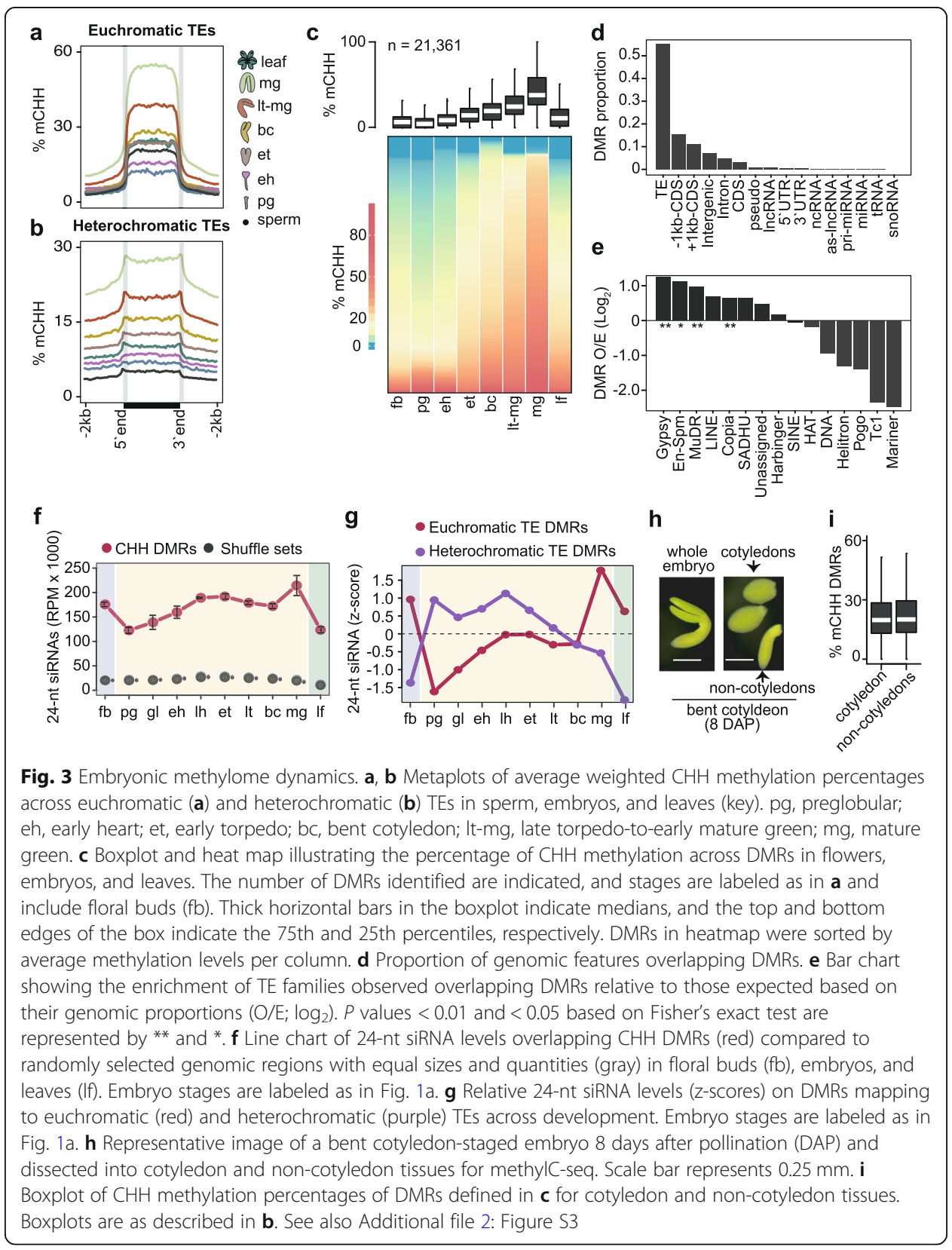


Table S4, Additional file 2: Figure S3d). Consistent with the genome-wide CHH methylation dynamics described above, embryonic $\mathrm{CHH}$ DMRs were hypomethylated in preglobular embryos, progressively methylated until embryo maturation, and then were sharply reduced in leaves and floral buds (Fig. 3c and Additional file 2: Figure S4A). Moreover, these embryonic DMRs were observed on various TE families especially Gypsy and Copia LTR retrotransposons, as well as MuDR and En-Spm family DNA TEs (Fig. 3d, e). The enrichment of mCHH DMRs across various TE families was generally consistent with the corresponding levels of embryonic 24-nt siRNAs (Fig. 3e and Additional file 2: Figure S2F). To examine the relationships between siRNAs and methylation further, we quantified 24-nt siRNA levels on mCHH DMRs across embryogenesis. Compared to randomized controls, CHH DMRs were highly enriched for 24-nt siRNAs (Fig. 3f). siRNAs overlapping euchromatic TE DMRs were lowest in early embryos, peaked at maturation and were also abundant in floral buds and leaves. In contrast, siRNAs overlapping heterochromatic TE DMRs were highly abundant at early-to-middle stages of embryogenesis and then strongly reduced in late-embryonic stages, leaves, and floral buds (Fig. 3g). Therefore, large-scale changes of DNA methylation occur on both euchromatic and heterochromatic TEs during embryogenesis and are associated with 24-nt siRNAs.

Notably, both euchromatic and heterochromatic TEs were hypermethylated in mature embryos relative to earlier stages and post-embryonic tissues (Fig. 3a-c and Additional file 2: Figure S3B). Because the proportion of embryonic tissue composed of cotyledons also increases during embryo development, we tested whether the progressively increasing levels of embryonic $\mathrm{CHH}$ methylation could be merely due to increased proportions of cotyledon tissues in embryos as they develop. Namely, we dissected cotyledon and non-cotyledon tissues from bent-cotyledon-staged embryos, and profiled their methylomes (Fig. 3h). Both cotyledon and non-cotyledon tissues were similarly hypermethylated on DMRs indicating that hypermethylation occurs throughout late-staged embryos and is not confined to the terminally differentiated cotyledons (Fig. 3i). Interestingly, the $\mathrm{CHH}$ hypermethylation observed in mature embryos resembled the hypermethylation reported in root columella cells and pollen vegetative nuclei $[30,50]$. Similar to these specific cell-types, mature embryos have also exited the cellcycle, which is further supported by increased and decreased levels of transcripts encoding negative and positive regulators of the cell-cycle, respectively (Additional file 2: Figure S3E) [51]. Therefore, CHH hypermethylation of mature embryos, as well as root columella cells and vegetative nuclei, is associated with cell cycle dormancy or exit.

\section{Small RNA-directed methylation of transposons during embryogenesis}

To test whether 24-nt siRNAs derived from euchromatic and heterochromatic TEs were necessary for progressive TE methylation during embryogenesis, we performed methylC-seq on 24-nt siRNA-deficient early heart (4 DAP) and bent-cotyledon embryos (8 DAP), as well as leaves and floral buds (Additional file 1: Table S1). NRPD1A encodes the largest subunit of RNA polymerase IV (Pol IV) [16], and accordingly, 24-nt siRNAs overlapping euchromatic and heterochromatic TEs were nearly eliminated in nrpd1a-3 mutants (Fig. 4a, c). Nearly all euchromatic TEs were completely hypomethylated in nrpd1a embryonic and post-embryonic tissues (Fig. 4b and Additional file 2: 


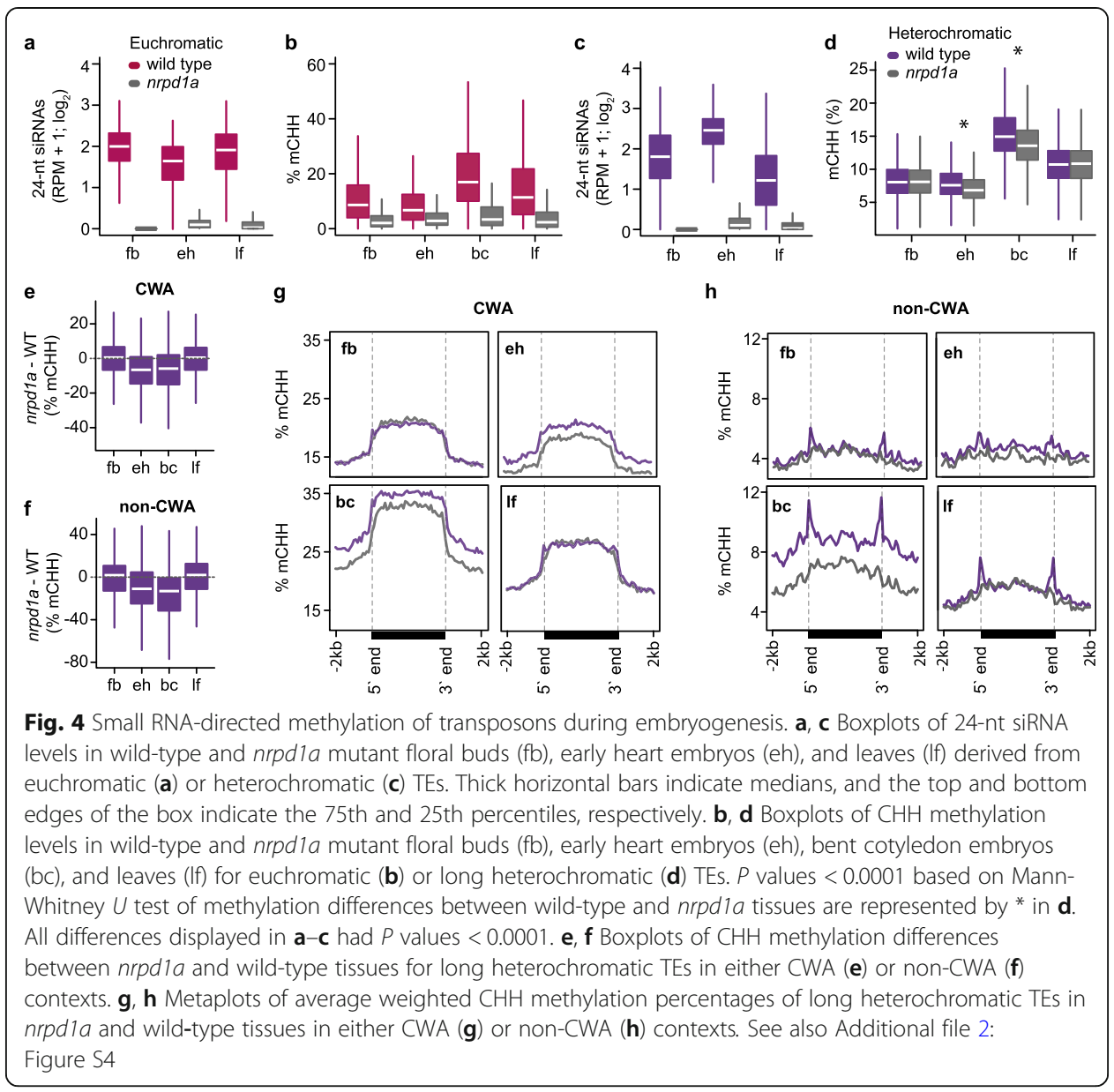

Figure S4). Because the 5729 heterochromatic TEs classified based on their embryonic siRNA dynamics also included short TEs methylated by the RdDM pathway in postembryonic tissues (Additional file 2: Figure S2D, E), we partitioned heterochromatic TEs into either short ( $\leq 723 \mathrm{bp}$ ), medium (724-2114 bp), or long (>2114) and examined their methylation levels in nrpd1a tissues (Fig. $4 \mathrm{~d}$ and Additional file 2: Figure S5A-C). Consistent with previous observations from post-embryonic tissues [26, 27, 43], $\mathrm{CHH}$ methylation of short and medium TEs was significantly reduced compared to wild type in all nrpd1a tissues tested including embryos (Additional file 2: Figure S5B, C). In contrast, long heterochromatic TEs were globally unaffected in nrpdla post-embryonic tissues, but significantly hypomethylated in nrpd1a mutant embryos relative to wild type (Fig. 4d). Methylation of long heterochromatic TEs is thus partially dependent on siRNAs in embryonic, but not post-embryonic tissues.

Long heterochromatic TEs are methylated by CMT2 in post-embryonic tissues, and their highly condensed chromatin states were proposed to inhibit siRNA-directed DRM2-mediated methylation [26, 27, 43, 52]. CHH methylation can be classified as CWA (W $=\mathrm{A}$ or $\mathrm{T}$ ) or non-CWA. DRM2 methylates CWA and non-CWA sites, and CMT2 preferentially methylates CWA nucleotides [53-55]. Only the edges of long heterochromatic TEs were hypomethylated in non-CWA contexts of post-embryonic nrpd1a mutant tissues relative to wild type. However, both edges and bodies of long heterochromatic TEs were hypomethylated in all $\mathrm{CHH}$ contexts in early heart and 
especially bent-cotyledon nrpd1a embryos compared to wild type (Fig. 4g, h). Therefore, 24-nt siRNAs originating from both bodies and edges of long heterochromatic TEs rapidly increase in early embryos and are partially required for TE methylation.

\section{Chromatin regulates small RNA transcription}

Heterochromatin prevents access to de novo methyltransferases [27, 56], and thus, it may also impede Pol IV access and resulting siRNA transcription. Consistent with relatively low transcript levels of heterochromatin-promoting factors in early embryos [57], reduction of DAPI-stained chromocenters and enlarged nuclei in zygotes compared to somatic tissues indicated that zygotic chromatin is decondensed (Fig. 5a, b and Additional file 2: Figure S6A). A marked increase in zygotic nucleoli size, as well as the co-localization of hundreds of $5 \mathrm{~S}$ rRNAs and heterochromatic TEs in centromeric regions (Copenhaver, 1999; Simon et al., 2018), further suggested that heterochromatic TEs could be decondensed in early embryos (Fig. 5b, c) Therefore, post-fertilization heterochromatin decondensation, potentially associated with rRNA production, may permit Pol IV accessibility to heterochromatic TEs and corresponding transcription of 24-nt siRNA precursors soon after fertilization. We then used a more experimentally tractable post-embryonic tissue to test how chromatin may generally regulate siRNA production.

Because linker histone 1 (H1) inhibits RNA polymerases from binding to chromatin $[60,61]$, and its depletion results in the loss of both chromocenters and chromatin compaction [59, 62, 63], we tested whether decreased H1 levels during postembryogenesis were sufficient to increase siRNA biogenesis throughout long heterochromatic TEs similar to what we observed in embryos. That is, we performed sRNAseq on leaves carrying null mutations in the two expressed H1.1 and H1.2 isoforms (i.e., $h 1.1-1 / h 1.2-1$ or $h 1$ mutants) and found that 24-nt siRNAs from heterochromatic TEs were significantly increased by more than 3.7 -fold and were predominantly derived from TE bodies (Fig. 5d: Additional file 2: Figure S6B). Strikingly, hierarchical clustering of TE-derived 24-nt siRNAs demonstrated that $h 1$ leaf siRNA populations were more similar to siRNA populations from wild-type mature embryos instead of leaves indicating that $\mathrm{H} 1$ depletion was sufficient to induce an embryo-like siRNA population in a post-embryonic tissue (Additional file 2: Figure S6C).

To investigate the effects of $\mathrm{H} 1$ depletion on TE-derived siRNAs in more detail, we ranked euchromatic and heterochromatic TEs based on their 24-nt siRNA levels in $h 1$ mutant leaves and found that increased 24-nt siRNA levels from long heterochromatic TEs in $h 1$ mutants were positively correlated with TE length, $\mathrm{CHH}$ methylation, and H1 occupancy (Fig. 5e-g and Additional file 2: Figure S6D). Because TEs with increased 24-nt siRNAs in $h 1$ mutants were also enriched for HISTONE 3 LYSINE 9 dimethylation (H3K9me2) (Fig. 5h), we tested whether loss of H3K9me2 can also affect heterochromatic siRNA production. We examined the levels of siRNAs from long heterochromatic TEs in leaves triple mutant for SU(VAR)3-9 HOMOLOG 4/5/6 histone methyltransferases (suvh4/5/6), which are deficient in H3K9me2 levels (Additional file 1: Table S1) [43]. Long heterochromatic TEs produced only 1.2-fold more 24-nt siRNAs in suvh4/5/6 leaves compared to wild type, and the global 24-nt siRNA populations were similar to wild type (Additional file 2: Figure S6B, C). These results suggest that 


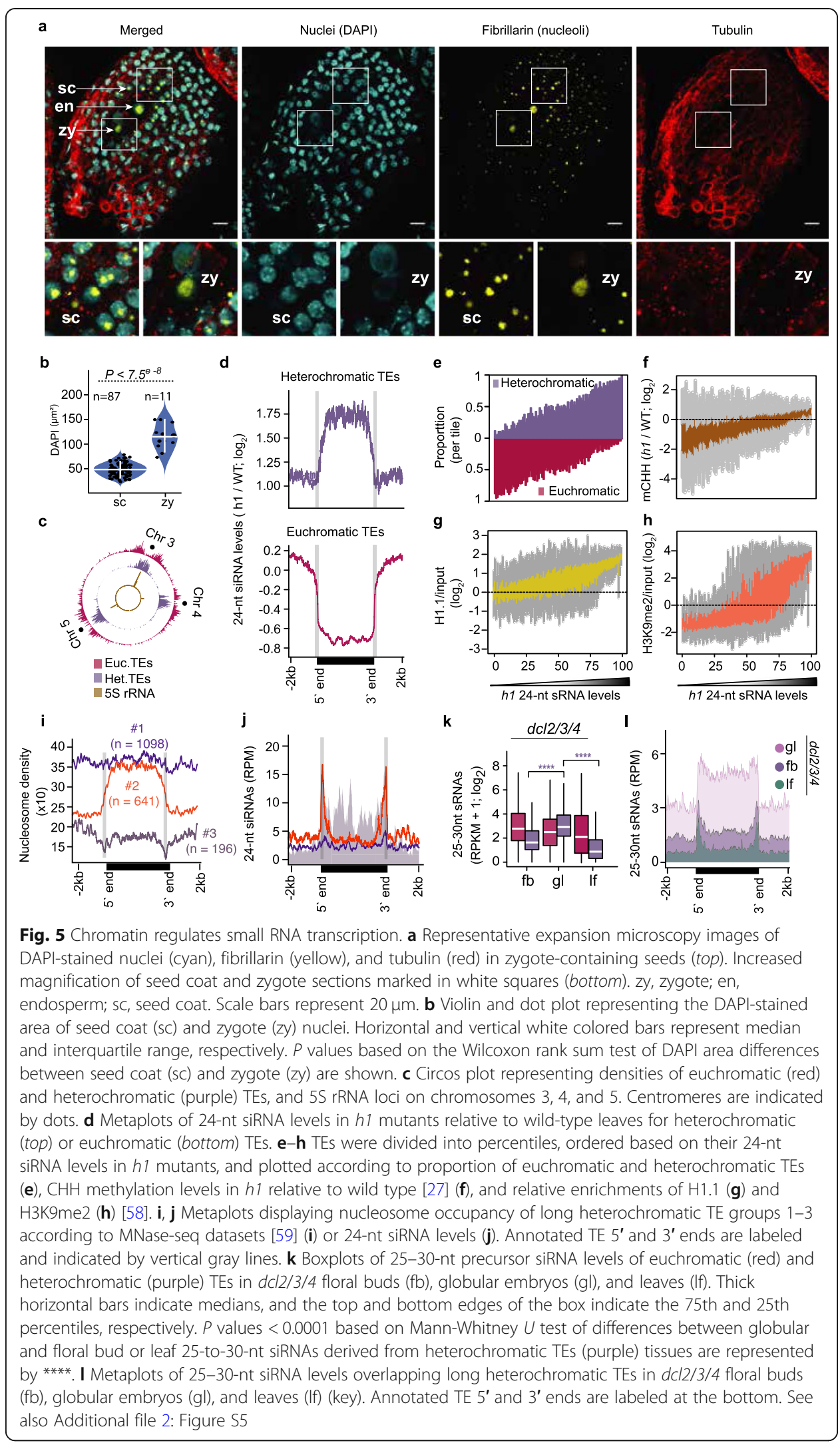


reducing heterochromatin, rather than H3K9me2 marks associated with heterochromatin, is sufficient for siRNA production from long heterochromatic TEs. Accordingly, H1 promotes nucleosome occupancy on heterochromatic regions [58, 59], and we found that heterochromatic TEs were enriched for $\mathrm{H} 1$ (Fig. $5 \mathrm{~g}$ and Additional file 2: Figure $\mathrm{S6D}$ ) and had reduced nucleosome occupancy in $h 1$ mutants (Additional file 2: Figure $\mathrm{S6F}$ ). Our results indicate that depletion of $\mathrm{H} 1$ is sufficient to decrease nucleosome occupancy of heterochromatic TEs, as well as increase corresponding siRNA and $\mathrm{CHH}$ methylation levels.

Because 24-nt siRNAs were enriched on the bodies of long heterochromatic TEs in h1 mutants with reduced chromatin compaction (Fig. 5d and Additional file 2: Figure S6B), we next examined the relationships between nucleosome occupancy and 24-nt siRNA levels of long heterochromatic TEs in wild-type leaves. We employed an iterative $k$-means clustering approach to generate three groups of long heterochromatic TEs based on their nucleosome occupancy using publicly available micrococcal nuclease sequencing data [59] (Fig. 5i). Group 1 comprised 1098 TEs (56.7\% of total) that had high densities of nucleosomes and were devoid of 24-nt siRNAs throughout their lengths (Fig. 5i, j). Group 2 contained 641 TEs (33.1\% of total) and had low and high nucleosome occupancy over the edges and bodies, respectively, and were enriched for 24-nt siRNAs only on the edges (Fig. 5i, j). Group 3 consisted of only 196 TEs (10.2\% of total) and had very low nucleosome levels, but abundant 24-nt siRNAs, on both their edges and bodies similar to euchromatic TEs (Fig. 5i, j). Altogether, these results suggest that increased nucleosome occupancy restricts RNA Pol IV activity, and thus, chromatin states alone appear to explain 24-nt siRNA production from TEs.

RNA Pol IV transcribes $\sim 25$-to-40-nt RNAs that are co-transcriptionally converted to double-stranded RNAs by RNA-dependent RNA Polymerases and rapidly processed into 23-nt/24-nt duplexes by DICER-LIKE (DCL) endoribonucleases [17, 18, 22]. These transient Pol IV-dependent 24-nt siRNA precursors can be robustly detected in $d c l 2 / 3 /$ 4 mutants [17, 18, 64], and thus, 24-nt siRNA precursor levels indicate Pol IV transcriptional activities. We performed sRNA-seq on $d c l 2 / 3 / 4$ flowers, globular embryos, and leaves and compared levels of 24-nt siRNA precursors from euchromatic and heterochromatic TEs (Additional file 1: Table S1). Compared to leaves and flowers, we respectively detected 11 -fold and 3.8-fold significantly more 24-nt precursors from heterochromatic TEs in $d c l 2 / 3 / 4$ early embryos (Fig. 5k). Importantly, the 24-nt siRNA precursors mostly originated from the bodies of long heterochromatic TEs in embryos, but were strongly reduced in floral buds and leaves (Fig. 5l). Together with the observations that 24-nt siRNAs were also enriched on the bodies of long heterochromatic TEs in early embryos and $h 1$ mutant leaves (Figs. 2c, and 5 d), our results are consistent with a model whereby decompaction of heterochromatin in early embryos and $h 1$ mutant leaves permits Pol IV access and transcriptional activities to produce 24-nt siRNAs.

\section{Homeostasis of transposon-derived siRNAs}

In contrast to heterochromatic TEs, we found 2.7-fold significantly less 24-nt siRNAs from euchromatic TEs in $h 1$ leaves compared to wild type, which was also associated with their $\mathrm{CHH}$ hypomethylation (Fig. 5d, f and Additional file 2: Figure S6B). Unlike heterochromatic TEs, euchromatic TEs were lowly enriched for $\mathrm{H} 1$ in wild-type leaves 
and nucleosome occupancy was further reduced in $h 1$ mutants (Fig. $5 \mathrm{~g}$ and Additional file 2: Figure S6D, F). Therefore, siRNA depletion from euchromatic TEs is likely an indirect consequence of sequestering Pol IV to accessible heterochromatic TEs. Moreover, we observed the greatest enrichment of siRNAs derived from heterochromatic compared to euchromatic TEs at the preglobular stage of embryogenesis, which is the earliest post-fertilization sRNA-seq dataset available (Fig. 6a). This was reduced during mid-embryogenesis and then further decreased to almost post-embryonic levels during maturation (Fig. 6a). Together with our siRNA precursor analysis (Fig. 5k, l), this indicates that Pol IV is more efficiently recruited to heterochromatic TEs compared to euchromatic TEs during the initial stages of embryogenesis. The enrichment of heterochromatic TE-derived 24-nt siRNAs in preglobular embryos surpassed what we observed in $h 1$ mutant leaves (Fig. 6a), suggesting that reduced nucleosome occupancy alone does not fully account for the extreme enrichment of heterochromatic siRNAs in preglobular embryos.

Based on available sRNA-seq datasets, euchromatic, but not heterochromatic, TEderived 24-nt siRNAs were substantially reduced in mutants deficient in CG and CHH methylation (Fig. 6b and Additional file 2: Figure S7) [21, 43, 69]. Therefore, low CHH

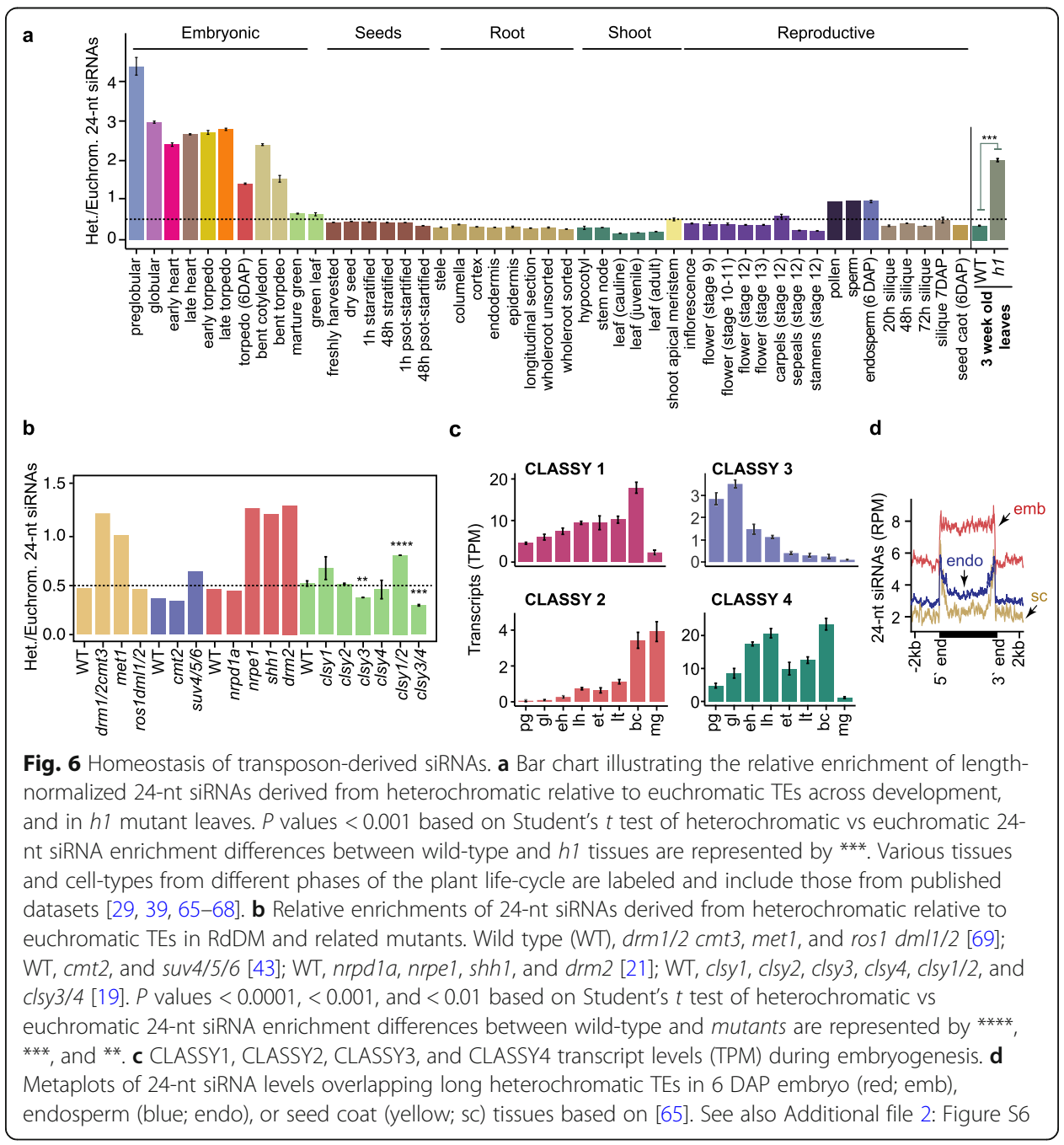


methylation in preglobular embryos (Fig. 3a, b) may reduce methylation-dependent feed-back loops that facilitate production of siRNAs from euchromatic TEs in preglobular stages. CLASSY (CLSY) chromatin remodeling factors also promote 24-nt siRNA production: CLSY $1 / 2$ and CLSY $3 / 4$ help recruit Pol IV to euchromatic and heterochromatic regions, respectively (Fig. 6b) [19, 21, 43, 69]. Dynamic chromatin states and corresponding establishment of methylation/CLSY-dependent transcription of siRNA precursors likely contribute to the unique siRNA populations observed in embryos.

Remarkably, the relative amounts of euchromatic and heterochromatic TE-derived siRNAs remained stable throughout post-embryonic development (Fig. 6a). Root columella and pollen vegetative cells are depleted for $\mathrm{H} 1$ [50,70], but were not depleted for euchromatic siRNAs (Fig. 6a). This may be due to these terminally differentiated celltypes being derived from a single mitotic division, and thus, they may retain the ability to recruit Pol IV to euchromatic TEs. For example, these cell types are $\mathrm{CHH}$ hypermethylated [30, 50], and thus, $\mathrm{CHH}$ methylation-dependent positive feedback loops on euchromatic TEs may counteract the loss of H1. Importantly, TE-derived siRNA populations in embryos were distinct from endosperm and seed-coat tissues (Fig. 6d). Consistent with chromatin states primarily regulating siRNA production, the endosperm has reduced cell division compared to embryos by 6 DAP [71]. Accordingly, endosperm siRNAs overlapped mostly edges, but not bodies, of heterochromatic TEs typical of other non-embryonic populations (Fig. 6d). Similar to pollen, but in contrast to other non-embryonic tissues, endosperm had similar levels of siRNAs from heterochromatic and euchromatic TEs (Fig. 6a). Because nearly equal siRNA levels from euchromatic and heterochromatic siRNAs were also observed in met1 mutants (Fig. 6b and Additional file 2: Figure S7), this balance may be due to loss of CG methylationdependent euchromatic siRNA production in CG hypomethylated pollen vegetative nuclei and endosperm [32]. Altogether our data indicate that the homeostasis of 24-nt siRNA production from euchromatic and heterochromatic TEs are affected by dynamic chromatin states including, but not restricted to, those associated with early embryogenesis.

\section{Discussion}

Although siRNAs direct faithful re-establishment of methylation genome-wide across generations [72], the dynamics of embryonic siRNAs and how they contribute to the nascent epigenome have not been reported. In this study, we demonstrated that thousands of TEs produce exceptionally high levels of 24-nt siRNAs in embryos (Fig. 1) and can be classified into two distinct groups based on their developmental dynamics (Fig. 2). siRNAs from euchromatic TEs gradually increase to post-embryonic levels during embryogenesis and are constitutively required to direct TE methylation in embryonic and post-embryonic tissues (Fig. 4). In contrast, heterochromatic TEs produce a burst of siRNAs soon after fertilization, and specifically during embryogenesis, to help establish TE methylation de novo, which is then maintained independent of siRNAs during post-embryogenesis (Fig. 4) [26, 27, 43]. Interestingly, the levels of siRNAs from these euchromatic and heterochromatic bipartite-classified TEs are regulated according to their chromatin states (Fig. 5). Decondensed chromatin permits transcription of 24nt siRNAs, and this contributes to cell autonomous homeostatic control mechanisms that normalize chromatin states. 
We propose a three-phase model for how chromatin states, and resulting siRNA dynamics, help shape the nascent epigenome (Fig. 7). After fertilization, zygotic chromatin is decondensed and this appears to be associated with transcriptional activation of rRNAs, including hundreds of $5 \mathrm{~S}$ rRNA loci that co-localize with heterochromatic TEs near centromeric regions (Fig. 5a, c). Arabidopsis zygotes require de novo synthesis of gene products directly after fertilization [57, 73], and genes involved in rRNA biogenesis produce high levels of transcripts in preglobular embryos relative to later stages [40]. In contrast to Arabidopsis, maternally donated proteins drive early embryogenesis in Xenopus and $\mathrm{H} 1$ dynamics mediate transcriptional activation of rRNA loci in oocytes and their silencing in somatic tissues $[74,75]$. In pollen vegetative cells, decondensation of rRNA loci can also permit their transcription and concomitant cell growth [76] and may be required for the rapid cell divisions in early endosperm, which also have enlarged nucleoli (Fig. 5a). Therefore, reduced heterochromatin in a variety of cell types, including those producing large amounts of protein such as early embryos, endosperm, and pollen vegetative cells, may be permissive for Pol IV-mediated transcription of siRNA precursors from TEs that are typically in a deep heterochromatic state during other developmental phases (Fig. 7; phase 1). Consistent with decondensation of heterochromatin facilitating de novo production of sRNAs, Pol IV-dependent 24-nt siRNAs were sharply increased throughout heterochromatic TEs in $h 1$ mutant leaves with reduced heterochromatin (Fig. 5d and Additional file 2: Figure S6). Production of siRNAs from euchromatic TEs is delayed relative to those from heterochromatic TEs (Fig. 6a). Because euchromatic, but not heterochromatic, TEs require $\mathrm{CHH}$ methylation and CLSY1/2 chromatin remodelers to produce full siRNA levels (Fig. 6b) [19, 21, 43, 69], both of which are gradually increased during embryogenesis (Figs. 3a-c and 6c), the developmental time-lag in euchromatic compared to heterochromatic siRNA production may also be partially due to the delay establishing methylation/CLSYdependent positive feedback loops during early embryogenesis.

Embryos divide rapidly through the bent cotyledon stage [77] and dynamic chromatin condensation and decondensation associated with such increased cell division [78, 79] likely allows access of Pol IV to both heterochromatic and euchromatic TEs (Fig. 7;

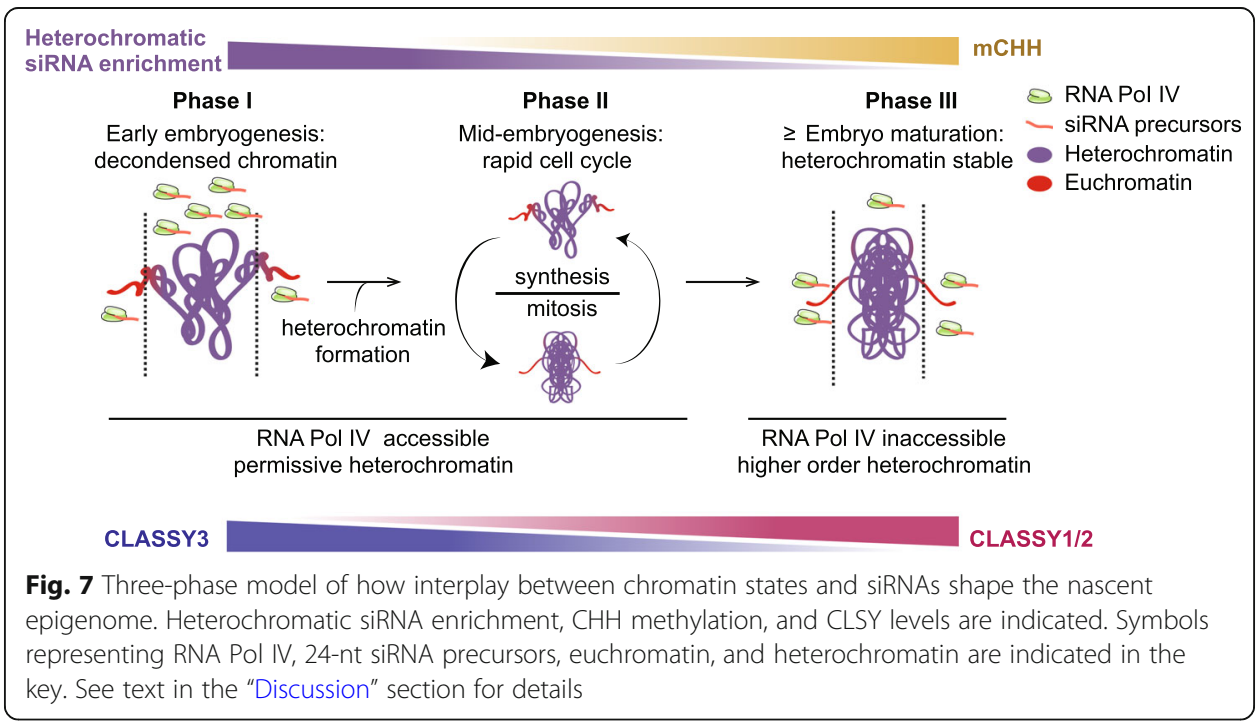


Phase 2). Production of embryonic siRNAs from heterochromatic relative to euchromatic TEs is steady between the globular and bent cotyledon stages. Heterochromatic TE-derived siRNAs are rapidly reduced upon maturation when heterochromatin becomes highly condensed [80] and euchromatic domains containing genes encoding seed storage and oil body biogenesis proteins are transcriptionally activated [40] (Fig. 6a). As a consequence, Pol IV access to heterochromatic TEs is likely greatly reduced, and this results in more Pol IV being readily available to produce siRNAs from euchromatic TEs (Fig. 7; Phase 3). Consistently, we observed a burst of siRNAs from euchromatic TEs, and their associated hyper methylation, at the mature stage (Fig. 2a, b). Heterochromatic TEs also become hypermethylated at the mature stage, which appears to be largely independent of siRNAs, but rather dependent on CMT2 as is typical of subsequent post-embryonic development (Fig. 4). Therefore, CHH hypermethylation throughout mature embryos may largely be a consequence of the rapid shift of chromatin states upon maturation.

Similar to $h 1$ mutants $[58,59]$, heat stress also causes reduced nucleosome occupancy and decondensed heterochromatin [81, 82]. Moreover, we classified the heat-activated ONSEN/AT5TE15240 as a class B/heterochromatic TE based on its siRNA dynamics, and ONSEN transcription and transposition are greatly enhanced in RdDM-defective mutants [83]. Interestingly, 24-nt siRNAs were increased throughout the body of ONSEN TEs after heat stress, and this was further enhanced in rapidly dividing undifferentiated calli [84], which has increased chromatin accessibility in rice [84, 85]. Based on these results, as well as our previous observation that heat-stress-related genes are significantly enriched in preglobular embryos [40], we suggest that the chromatin dynamics caused by heat stress and subsequent recovery are analogous to what we observed in early embryos. That is, heat stress and fertilization may decrease nucleosome occupancy across heterochromatic TEs, which enables Pol IV-mediated siRNA production and subsequent reconstitution of proper heterochromatin to help limit TE mobilization in the genome. Strong upregulation of TE-specific endo-siRNAs and piwiinteracting RNAs observed in H1-depleted Drosophila [86] suggests that H1-dependent regulation of chromatin states may also facilitate heterochromatic small RNA transcription in animals.

\section{Conclusions}

Reprogramming of heterochromatin during early embryogenesis occurs in diverse metazoa including flies, mammals, worms, and zebrafish [8-12], and we observed that $\mathrm{CHH}$ methylation is also reprogrammed during early plant embryogenesis (Fig. 3). For example, $\mathrm{CHH}$ methylation was essentially lost on the bodies of heterochromatic TEs in sperm and subsequently fully re-established by both siRNA-dependent and siRNAindependent pathways (Fig. 4). Reduced heterochromatin in early animal embryos has been associated with increased developmental potential [87, 88], and similar relationships have also been observed in plants including reprogramming associated with plant regeneration and heat-stress induced somatic embryogenesis [89-95]. However, decreased heterochromatin would also increase the risk of TE mobilization and resulting mutations, and this could be especially dangerous in plant zygotes because they are the precursors of all cell types including the gametes. Our results indicate that embryos produce 24-nt siRNAs according to their chromatin states including those that are 
permissible for Pol IV transcription soon after fertilization. These de novo produced 24-nt siRNAs direct re-methylation of both euchromatic and heterochromatic TEs in the new generation. Therefore, decondensed chromatin permits transcription of early embryonic siRNAs to help promote cell-autonomous TE silencing. More generally, reduced heterochromatin due to sharp increases in rRNA production requirements during growth (e.g., early embryos, endosperm, and pollen vegetative cells), and perhaps in response to external cues such as heat stress, enables the synthesis and functions of sRNAs that can help reconstitute proper chromatin states.

\section{Methods}

Plant material and growth conditions

All genotypes were in the Columbia-0 (Col-0) Arabidopsis thaliana background including $d c l 2 / 3 / 4$ mutants composed of $d c l 2-1$, dcl3-1 and dcl4-2t [96], h1.1-1/h1.2-2 [27], nrpd1a-3 [97], and suv4/5/6 [98]. Plants were grown in a climate-controlled growth chamber at 20 to $22{ }^{\circ} \mathrm{C}$ under a 16-h light/8-h dark cycle with incandescent lights at 130 to $150 \mu \mathrm{mol} / \mathrm{m}^{2} / \mathrm{s}$.

\section{Embryo isolation and nucleic acid extraction}

Embryos were dissected from siliques either 3 days after pollination (DAP) (preglobular), 4 DAP (early heart/transition), or 8 DAP (bent cotyledon). Siliques were opened with forceps and seeds were collected in 2-ml Eppendorf tubes containing nucleasefree water and kept on ice. Seeds were then crushed with pestles, and embryos were selected under an inverted microscope using a microcapillary tube. Isolated embryos, as well as cotyledon and non-cotyledon portions of bent-cotyledon embryos, were thoroughly and serially washed $4 \times$ with nuclease-free water and stored at $-80^{\circ} \mathrm{C}$. RNA was isolated as previously described [39, 99]. Genomic DNA was extracted from $\geq 50 \mathrm{em}$ bryos per stage, floral buds, and leaves using Quick-DNA ${ }^{\mathrm{Tm}}$ Micro prep Kit (Zymo D3020) according to the manufacturer's recommendations.

\section{Small RNA profiling}

sRNA-seq libraries were generated as previously described [39]. Briefly, total RNA from each sample was size selected for 18 to 30-nucleotide RNAs using denaturing polyacrylamide-urea gels. Size-selected RNA was used to ligate adapters and synthesize cDNA with the NEBNext Multiplex Small RNA Library Prep Set for Illumina kit (cat. no E7300; New England Biolabs) according to the manufacturer's recommendations. Various numbers of PCR cycles were used to amplify cDNAs: 18, 20, 22, and 24 PCR cycles for globular and 14, 16, 18, and 20 PCR cycles for early heart and 3-week-old leaf samples. Final PCR amplicons were initially run on a $90 \%(\mathrm{v} / \mathrm{v})$ formamide/8\% (w/v) acrylamide gel for $30 \mathrm{~min}$ at $5 \mathrm{~W}$, followed by $30 \mathrm{~W}$ for $\geq 2 \mathrm{~h}$, and stained with SYBR Gold (1:10,000; Thermo Fisher Scientific). PCR amplicons between 137 and 149 bp corresponding to 18- to 30-nucleotide sRNAs with adapters, respectively, were inspected under a UV transilluminator, and amplicons with non-saturated signals generated from PCR cycles were gel-purified. Gel-purified small RNA libraries were resuspended in $15 \mu \mathrm{L}$ of Elution Buffer (Qiagen). Finally, small RNA libraries were quality checked for the expected size range with Agilent High sensitivity NGS fragment Kit (DNF-474- 
1000) and were sequenced on a HiSeq 2500 instrument (Illumina) in 50-base singleend mode.

\section{Small RNA sequencing analysis}

Small RNA-seq library datasets generated in this study or downloaded from NCBI's Sequence Read Archive (SRA) were subjected to the same small RNA analysis pipeline. First, raw fastq files were adapter trimmed with Cutadapt [100] and sequences between 18 and 30 bases in length, and that contained an adapter were retained. The trimmed sequences were then aligned to the Arabidopsis thaliana TAIR10 genome [101] with STAR [102] requiring zero mismatches and allowing up to 100 multiple end-to-end alignments. Multi-mapping reads from aligned SAM files were re-assigned with a "richget-richer" algorithm using the custom python script "readmapIO.py" as described previously [103]. Resulting output bedFiles were then sorted, condensed, and normalized for total genome-matching reads. The BEDtools [104] map function was then used to quantify the sum of the normalized reads per million (RPM) mapping to TAIR10 annotated Transposable elements (TEs).

For the model-based clustering of transposon-derived 24-nt siRNAs, mean RPM of 24-nt siRNAs from biological triplicates of floral bud, embryonic, and leaf samples mapping to TEs were calculated and used as input for $R$ library Mclust [42] to identify the optimal Gaussian mixture model (GMM). By employing Mclust function mclust$B I C(., G=s e q(2,20), b y=2)$ in sequential increments of two until twenty components, we identified the VEV (Variable volume, Equal shape, Variable orientation) ellipsoidal distribution model to be optimal with the minimum number of components (i.e., eight) containing maximum Bayesian Information Criterion (BIC). Finally to yield eight transposon clusters with VEV ellipsoidal distribution model, the Mclust., $G=8$, modelNa$\left.m e s=" V E V^{\prime \prime}\right)$ function was applied on mean TE-derived 24-nt siRNAs.

Principal component analysis of 24-nt siRNAs was performed with the $\mathrm{R}$ prcomp function using default parameters. Hierarchical clustering of transposon-derived 24-nt siRNAs was performed by calculating Euclidean distances between samples and the distance matrix was subjected to the $\mathrm{R}$ function hclust("," complete"). Heatmaps and metaplots of TE-derived siRNAs were generated with deepTools [105]. Briefly, a matrix containing normalized 24-nt siRNA scores per genome regions for tissue types or genotypes were generated (computeMatrix scale-regions -bs 5 -m 4000 -b 2000 - 2000 --averageTypeBins mean). The obtained matrix was used to generate heatmaps (deepTools plotHeatmap) or metaplots (deepTools plotProfile). For Fig. 5, regions without siRNA signals were removed and the remaining genomic regions were used to calculate matrix containing nucleosome signal and 24-nt siRNA levels. This matrix then served as input to employ Iterative K-means clustering with deepTools function plotProfile --kmeans.

\section{DNA methylation profiling}

MethylC-seq libraries were generated using post-bisulfite adapter tagging (PBAT) to avoid the bisulfite-induced loss of intact sequencing templates as described [48] with the following modifications. Briefly, genomic DNA was subjected to bisulfite treatment for 200 min with EZ DNA Methylation-DirectTM Kit (Zymo D5020). Bisulfite-treated 
DNA was then preamplified for two cycles with primers (5'-CCCTACACGACGCTCT TCCGATCTNNNNNN-3') containing random hexamers and purified using the Zymo DNA Clean and Concentrator kit. Adaptor primers (5' -CAGACGTGTGCTCTTCCG ATCTNNNNNN-3') were added to preamplified products and then amplified for 12 PCR cycles with indexing primers for Illumina sequencing. Methylome libraries were purified using Beckman Coulter AMPureXP DNA beads. Libraries quality checked for fragment length between 200 and $600 \mathrm{bp}$ were used for sequenced in single-read mode on an Illumina HiSeq2500 or Nextseq instrument.

\section{DNA methylation analysis}

Sequenced reads were quality filtered and trimmed using Trim Galore with default settings. In addition, the first six bases of each read were removed to exclude random hexamers from the pre-amplification step of library construction and to also reduce 5' methylation-bias (m-bias). Reads were aligned against the C-to-T converted TAIR10 genome using Bismark in non-directional mode to original top strand (OT), original bottom strand (OB), complementary to OT (CTOT) and OB (CTOB) (bismark --non_directional -q --score-min L,0,-0.4) [106]. Aligned BAM files containing clonal duplicates were removed with function deduplicate_bismark -s --bam, and uniquely mapped reads were then used as input for the Methylpy software [107]. Weighted methylation rates at each covered cytosine was extracted using command methylpy call-methylation-state --pairedend FALSE. Bisulfite conversion rates were calculated using the unmethylated chloroplast genome or spiked-in unmethylated Lambda phage DNA controls (European Nucleotide Archive Accession Number J02459, Promega catalog number D1521). FASTQ files obtained from publicly available methylomes generated from sperm [32], early torpedo [49], mid-torpedo to early maturation [31], mature green [37] embryos, and H1 mutant tissues [27] were also processed in the similar manner; except only $5^{\prime}$ end nucleotides of the reads with $\mathrm{m}$-bias were removed and aligned in directional mode to OT and OB strands.

Differentially methylated regions (DMRs) were defined using Methylpy as described [36]. Briefly, biological replicates were pooled and differentially methylated sites (DMSs) were identified by the root mean square tests with false discovery rates $\leq 0.01$. Cytosine sites with $\geq 4$ overlapping reads were retained for all samples except for preglobular in which DMSs with $\geq 3$ overlapping reads were retained. Differentially methylated sites within 100-bp were collapsed into DMRs. CHH-DMRs were further filtered by discarding regions with $<4$ DMSs and methylation differences $<20 \%$. Using these parameters, DMRs were identified in all 10 pairwise combinations across embryonic samples (preglobular, early heart, early torpedo, bent cotyledon, mature green) and merged using the BEDtools merge function [104]. DMRs were used to calculate the weighted $\mathrm{CHH}$ methylation rate on all analyzed tissue types. $\mathrm{CHH}$ methylation metaplots for class A, B, and siRNA-deficient TEs were plotted using the R library Seqplots [108]: Body, upstream, and downstream regions of TEs were split into equal-sized bins, and the average weighted $\mathrm{mCHH}$ level for each bin was calculated and plotted.

\section{Expansion microscopy and DAPI quantification}

The expansion microscopy technique [109] optimized for Arabidopsis seeds was conducted as previously described [73]. Anti-Fibrillarin antibody (ab4566, Abcam) and 
anti-alpha Tubulin antibody (ab89984, Abcam) were used in 1:500 dilution as primary antibodies. Goat Anti-Mouse IgG H\&L (Alexa Fluor ${ }^{\circ} 488$ ) (ab150113, Abcam) and goat Anti-Chicken IgY H\&L (Alexa Fluor ${ }^{\circ}$ 555) (ab150170, Abcam) were used in 1:500 dilution as secondary antibodies. For each sample, a stack of nine images with $1-\mu \mathrm{m}$ intervals were recorded by ZEISS LSM700 with $25 \times$ oil objective and ZEN software at $1024 \times 1024$ resolution in 8 -bit. DAPI signals were excited by $405-\mathrm{nm}$ laser and passed through SP490 filters. Alexa488 signals were excited by $488-\mathrm{nm}$ laser and passed through BP490-635 filters. Alexa555 signals were excited by $555-\mathrm{nm}$ laser and passed through 560-1000-nm filters. Pinhole sizes were kept as 1 airy unit for each color, and color channels were scanned separately. FIJI software was used for image processing and nuclear size quantification. Each stack of images was first Z-projected on maximum intensity and then the nuclear areas were determined based on DAPI signals. The zygotic nuclei were distinguished from the endosperm nuclei according to position and tubulin staining patterns.

\section{Supplementary information}

Supplementary information accompanies this paper at https://doi.org/10.1186/s13059-020-02163-4.

Additional file 1: Table S1. Datasets and general mapping statistics.

Additional file 2: Figure S1. Characteristics of embryonic 24-nt siRNAs and their similarities across samples. Figure S2. siRNA dynamics and characteristics. Figure S3. Benchmarking low-input methylC-seq, methylomes and cell-cycle transcripts. Figure S4. Genome browser screenshots of 24-nt siRNAs and DNA methylation levels. Figure S5. Size-based partitioning of heterochromatic TEs and small RNA-directed methylation. Figure S6. TE-derived siRNA accumulation and association with chromatin. Figure S7. TE-derived siRNAs in methylation mutants.

Additional file 3: Table S3. Euchromatic and heterochromatic TE classifications.

Additional file 4: Table S4. Differentially methylated regions.

Additional file 5. Review history.

\section{Acknowledgements}

We thank the Vienna Biocenter Core Facilities GmbH (VBCF) Next Generation Sequencing and Plant Sciences Facilities for next-generation sequencing and plant growth chamber access, respectively, and the Institute of Molecular Pathology-Institute of Molecular Biology-Gregor Mendel Institute Molecular Biology Services for instrument access and support. We also thank Alexander Vogt for help in optimizing low-input methylC-seq library preparation; Anna Smolka for technical assistance; Patrick Hüther and Claude Becker for advice on methylation analysis; Zdravko Lorkovi, Michael Borg, and Frédéric Berger for sharing reagents; and Michael Schon, Balaji Enugutti, and other members of the Nodine lab for valuable input.

Peer review information

Kevin Pang was the primary editor on this article and managed its editorial process and peer review in collaboration with the rest of the editorial team.

Review history

The review history is available as Additional file 5.

\section{Authors' contributions}

R.K.P. and M.D.N. conceived the project; R.K.P. developed the methodology, implemented software used, and performed formal analysis; R.K.P., S.P., K.P., P.K., S.L., and M.D.N. conducted the experiments; R.K.P. and M.D.N. wrote and edited the article; M.D.N. supervised the project and acquired funding. The authors read and approved the final manuscript.

Funding

This work was supported by the European Research Council under the European Union's Horizon 2020 Research and Innovation Program (grant 637888 to M.D.N.).

\section{Availability of data and materials}

All sequencing data generated in this study are available at the National Center for Biotechnology Information Gene Expression Omnibus (NCBI GEO, https://www.ncbi.nlm.nih.gov/geo/) under accession number GSE152971 [110].

Publicly available next-generation sequencing data were downloaded from NCBI, GEO, and are listed along with general mapping statistics in Additional file 1: Table S1. The software code used for the sRNA, methylome, and transcriptome analysis including a nextflow pipeline is available at https://github.com/mnodine/Papareddy.2020 [111]. BigWig 
files of processed datasets generated either as part of this study or publicly available can be downloaded at https:// github.com/mnodine/Papareddy.2020/tree/master/processed_BigWigs [111].

Ethics approval and consent to participate

Not applicable.

\section{Consent for publication}

Not applicable.

\section{Competing interests}

The authors declare that they have no conflicts of interests.

Received: 10 April 2020 Accepted: 4 September 2020

Published online: 17 September 2020

\section{References}

1. Heitz E. Das heterochromatin der moose. I Jahrb Wiss Bot. 1928;69:762-818.

2. Grewal SIS, Jia S. Heterochromatin revisited. Nat Rev Genet. 2007;8:35-46.

3. Elgin SCR, Grewal SIS. Heterochromatin: silence is golden. Curr Biol. 2003;13:R895-8.

4. Huisinga KL, Brower-Toland B, Elgin SCR. The contradictory definitions of heterochromatin: transcription and silencing. Chromosoma. 2006:115:110-22.

5. Chan SW-L, Henderson IR, Jacobsen SE. Gardening the genome: DNA methylation in Arabidopsis thaliana. Nat Rev Genet. 2005:6:351-60

6. Bannister AJ, Kouzarides T. Regulation of chromatin by histone modifications. Cell Res. 2011;21:381-95.

7. Klemm SL, Shipony Z, Greenleaf WJ. Chromatin accessibility and the regulatory epigenome. Nat Rev Genet. 2019; 20:207-20.

8. Laue K, Rajshekar S, Courtney AJ, Lewis ZA, Goll MG. The maternal to zygotic transition regulates genomewide heterochromatin establishment in the zebrafish embryo. Nat Commun. 2019. https://doi.org/10.1038/ s41467-019-09582-3.

9. Wang C, Liu X, Gao Y, Yang L, Li C, Liu W, et al. Reprogramming of H3K9me3-dependent heterochromatin during mammalian embryo development. Nat Cell Biol. 2018;20:620-31.

10. Rudolph T, Yonezawa M, Lein S, Heidrich K, Kubicek S, Schäfer C, et al. Heterochromatin formation in Drosophila is initiated through active removal of H3K4 methylation by the LSD1 homolog SU(VAR)3-3. Mol Cell. 2007;26:103-15.

11. Mutlu B, Chen H-M, Moresco JJ, Orelo BD, Yang B, Gaspar JM, et al. Regulated nuclear accumulation of a histone methyltransferase times the onset of heterochromatin formation in C. elegans embryos. Sci Adv. 2018:eaat6224. https:// doi.org/10.1126/sciadv.aat6224.

12. Ahmed K, Dehghani H, Rugg-Gunn P, Fussner E, Rossant J, Bazett-Jones DP. Global chromatin architecture reflects pluripotency and lineage commitment in the early mouse embryo. PLoS One. 2010;5:e10531.

13. Ingouff M, Hamamura Y, Gourgues M, Higashiyama T, Berger F. Distinct dynamics of HISTONE3 variants between the two fertilization products in plants. Curr Biol. 2007:17:1032-7.

14. Ingouff M, Rademacher S, Holec S, Soljić L, Xin N, Readshaw A, et al. Zygotic resetting of the HISTONE 3 variant repertoire participates in epigenetic reprogramming in Arabidopsis. Curr Biol. 2010;20:2137-43.

15. Martienssen R, Moazed D. RNAi and heterochromatin assembly. Cold Spring Harb Perspect Biol. 2015;7:a019323.

16. Herr AJ, Jensen MB, Dalmay T, Baulcombe DC. RNA polymerase IV directs silencing of endogenous DNA. Science. 2005; 308:118-20.

17. Zhai J, Bischof S, Wang H, Feng S, Lee T-F, Teng C, et al. A one precursor one siRNA model for Pol IV-dependent siRNA biogenesis. Cell. 2015;163:445-55.

18. Blevins T, Podicheti R, Mishra V, Marasco M, Wang J, Rusch D, et al. Identification of Pol IV and RDR2-dependent precursors of 24 nt siRNAs guiding de novo DNA methylation in Arabidopsis. Elife. 2015;4:e09591.

19. Zhou M, Palanca AMS, Law JA. Locus-specific control of the de novo DNA methylation pathway in Arabidopsis by the CLASSY family. Nat Genet. 2018;50:865-73.

20. Law JA, Jacobsen SE. Establishing, maintaining and modifying DNA methylation patterns in plants and animals. Nat Rev Genet. 2010;11:204-20.

21. Law JA, Du J, Hale CJ, Feng S, Krajewski K, Palanca AMS, et al. Polymerase IV occupancy at RNA-directed DNA methylation sites requires SHH1. Nature. 2013;498:385-9.

22. Singh J, Mishra V, Wang F, Huang H-Y, Pikaard CS. Reaction mechanisms of Pol IV, RDR2, and DCL3 drive RNA channeling in the siRNA-directed DNA methylation pathway. Mol Cell. 2019;75:576-89.e5.

23. Zilberman D, Cao X, Jacobsen SE. ARGONAUTTE4 control of locus-specific siRNA accumulation and DNA and histone methylation. Science. 2003;299:716-9.

24. Wierzbicki AT, Haag JR, Pikaard CS. Noncoding transcription by RNA polymerase Pol IVb/Pol V mediates transcriptional silencing of overlapping and adjacent genes. Cell. 2008;135:635-48.

25. Cao X, Jacobsen SE. Locus-specific control of asymmetric and CpNpG methylation by the DRM and CMT3 methyltransferase genes. Proc Natl Acad Sci U S A. 2002;99(Suppl 4):16491-8.

26. Stroud H, Greenberg MVC, Feng S, Bernatavichute W, Jacobsen SE. Comprehensive analysis of silencing mutants reveals complex regulation of the Arabidopsis methylome. Cell. 2013;152:352-64.

27. Zemach A, Kim MY, Hsieh P-H, Coleman-Derr D, Eshed-Williams L, Thao K, et al. The Arabidopsis nucleosome remodeler DDM1 allows DNA methyltransferases to access H1-containing heterochromatin. Cell. 2013;153:193-205.

28. Drews GN, Koltunow AMG. The female gametophyte. Arabidopsis Book. 2011;9:e0155.

29. Slotkin RK, Vaughn M, Borges F, Tanurdzić M, Becker JD, Feijó JA, et al. Epigenetic reprogramming and small RNA silencing of transposable elements in pollen. Cell. 2009;136:461-72. 
30. Calarco JP, Borges F, Donoghue MTA, Van Ex F, Jullien PE, Lopes T, et al. Reprogramming of DNA methylation in pollen guides epigenetic inheritance via small RNA. Cell. 2012;151:194-205.

31. Hsieh T-F, Ibarra CA, Silva P, Zemach A, Eshed-Williams L, Fischer RL, et al. Genome-wide demethylation of Arabidopsis endosperm. Science. 2009;324:1451-4.

32. Ibarra CA, Feng X, Schoft VK, Hsieh T-F, Uzawa R, Rodrigues JA, et al. Active DNA demethylation in plant companion cells reinforces transposon methylation in gametes. Science. 2012;337:1360-4.

33. Feng X, Zilberman D, Dickinson H. A conversation across generations: soma-germ cell crosstalk in plants. Dev Cell. 2013; 24:215-25.

34. Mosher RA, Melnyk CW. siRNAs and DNA methylation: seedy epigenetics. Trends Plant Sci. 2010;15:204-10.

35. Jullien PE, Susaki D, Yelagandula R, Higashiyama T, Berger F. DNA methylation dynamics during sexual reproduction in Arabidopsis thaliana. Curr Biol. 2012;22:1825-30.

36. Kawakatsu T, Nery JR, Castanon R, Ecker JR. Dynamic DNA methylation reconfiguration during seed development and germination. Genome Biol. 2017;18:171.

37. Bouyer D, Kramdi A, Kassam M, Heese M, Schnittger A, Roudier F, et al. DNA methylation dynamics during early plant life. Genome Biol. 2017;18:179.

38. Lin J-Y, Le BH, Chen M, Henry KF, Hur J, Hsieh T-F, et al. Similarity between soybean and Arabidopsis seed methylomes and loss of non-CG methylation does not affect seed development. Proc Natl Acad Sci U S A. 2017;114:E9730-9.

39. Plotnikova A, Kellner MJ, Schon MA, Mosiolek M, Nodine MD. MicroRNA dynamics and functions during Arabidopsis embryogenesis. Plant Cell. 2019;31:2929-46.

40. Hofmann F, Schon MA, Nodine MD. The embryonic transcriptome of Arabidopsis thaliana. Plant Reprod. 2019;32:77-91.

41. Mi S, Cai T, Hu Y, Chen Y, Hodges E, Ni F, et al. Sorting of small RNAs into Arabidopsis argonaute complexes is directed by the $5^{\prime}$ terminal nucleotide. Cell. 2008;133:116-27.

42. Scrucca L, Fop M, Murphy TB, Raftery AE. mclust 5: clustering, classification and density estimation using Gaussian finite mixture models. R J. 2016;8:289-317.

43. Stroud H, Do T, Du J, Zhong X, Feng S, Johnson L, et al. Non-CG methylation patterns shape the epigenetic landscape in Arabidopsis. Nat Struct Mol Biol. 2014;21:64-72.

44. Cao X, Jacobsen SE. Role of the arabidopsis DRM methyltransferases in de novo DNA methylation and gene silencing Curr Biol. 2002;12:1138-44.

45. Qi Y, He X, Wang X-J, Kohany O, Jurka J, Hannon GJ. Distinct catalytic and non-catalytic roles of ARGONAUTE4 in RNAdirected DNA methylation. Nature. 2006;443:1008-12.

46. Wierzbicki AT, Ream TS, Haag JR, Pikaard CS. RNA polymerase V transcription guides ARGONAUTE4 to chromatin. Nat Genet. 2009;41:630-4.

47. Bies-Etheve N, Pontier D, Lahmy S, Picart C, Vega D, Cooke R, et al. RNA-directed DNA methylation requires an AGO4interacting member of the SPT5 elongation factor family. EMBO Rep. 2009;10:649-54.

48. Clark SJ, Smallwood SA, Lee HJ, Krueger F, Reik W, Kelsey G. Genome-wide base-resolution mapping of DNA methylation in single cells using single-cell bisulfite sequencing (scBS-seq). Nat Protoc. 2017;12:534-47.

49. Pignatta D, Erdmann RM, Scheer E, Picard CL, Bell GW, Gehring M. Correction: Natural epigenetic polymorphisms lead to intraspecific variation in Arabidopsis gene imprinting. Elife. 2015;4. https://doi.org/10.7554/eLife.08658.

50. Kawakatsu T, Stuart T, Valdes M, Breakfield N, Schmitz RJ, Nery JR, et al. Unique cell-type-specific patterns of DNA methylation in the root meristem. Nat Plants. 2016;2:16058.

51. Vandepoele K, Raes J, De Veylder L, Rouzé P, Rombauts S, Inzé D. Genome-wide analysis of core cell cycle genes in Arabidopsis. Plant Cell. 2002;14:903-16.

52. Lyons DB, Zilberman D. DDM1 and Lsh remodelers allow methylation of DNA wrapped in nucleosomes. eLife. 2017. https://doi.org/10.7554/elife.30674

53. Gouil Q, Baulcombe DC. DNA methylation signatures of the plant chromomethyltransferases. PLoS Genet. 2016;12:e1006526.

54. Li X, Harris CJ, Zhong Z, Chen W, Liu R, Jia B, et al. Mechanistic insights into plant SUVH family H3K9 methyltransferases and their binding to context-biased non-CG DNA methylation. Proc Natl Acad Sci U S A. 2018;115:E8793-802.

55. Wendte JM, Zhang Y, Ji L, Shi X, Hazarika RR, Shahryary Y, et al. Epimutations are associated with CHROMOMETHYLASE 3-induced de novo DNA methylation. Elife. 2019;8. https://doi.org/10.7554/eLife.47891.

56. Schoft VK, Chumak N, Mosiolek M, Slusarz L, Komnenovic V, Brownfield L, et al. Induction of RNA-directed DNA methylation upon decondensation of constitutive heterochromatin. EMBO Rep. 2009;10:1015-21.

57. Zhao P, Zhou X, Shen K, Liu Z, Cheng T, Liu D, et al. Two-step maternal-to-zygotic transition with two-phase parental genome contributions. Dev Cell. 2019;49:882-93.e5.

58. Choi J, Lyons DB, Kim MY, Moore JD, Zilberman D. DNA methylation and histone H1 jointly repress transposable elements and aberrant intragenic transcripts. Mol Cell. 2019. https://doi.org/10.1016/j.molcel.2019.10.011.

59. Rutowicz K, Lirski M, Mermaz B, Teano G, Schubert J, Mestiri I, et al. Linker histones are fine-scale chromatin architects modulating developmental decisions in Arabidopsis. Genome Biol. 2019;20:157.

60. Russanova VR, Driscoll CT, Howard BH. Adenovirus type 2 preferentially stimulates polymerase III transcription of Alu elements by relieving repression: a potential role for chromatin. Mol Cell Biol. 1995;15:4282-90.

61. Krishnakumar R, Gamble MJ, Frizzell KM, Berrocal JG, Kininis M, Kraus WL. Reciprocal binding of PARP-1 and histone H1 at promoters specifies transcriptional outcomes. Science. 2008;319:819-21.

62. Luger K, Mäder AW, Richmond RK, Sargent DF, Richmond TJ. Crystal structure of the nucleosome core particle at 2.8 a resolution. Nature. 1997;389:251-60.

63. Fyodorov DV, Zhou B-R, Skoultchi Al, Bai Y. Emerging roles of linker histones in regulating chromatin structure and function. Nat Rev Mol Cell Biol. 2018;19:192-206.

64. Yang D-L, Zhang G, Tang K, Li J, Yang L, Huang H, et al. Dicer-independent RNA-directed DNA methylation in Arabidopsis. Cell Res. 2016;26:1264.

65. Erdmann RM, Satyaki PRV, Klosinska M, Gehring M. A small RNA pathway mediates allelic dosage in endosperm. Cell Rep. 2017;21:3364-72.

66. Narsai R, Gouil Q, Secco D, Srivastava A, Karpievitch W, Liew LC, et al. Extensive transcriptomic and epigenomic remodelling occurs during Arabidopsis thaliana germination. Genome Biol. 2017;18:172. 
67. Breakfield NW, Corcoran DL, Petricka JJ, Shen J, Sae-Seaw J, Rubio-Somoza I, et al. High-resolution experimental and computational profiling of tissue-specific known and novel miRNAs in Arabidopsis. Genome Res. 2012;22:163-76.

68. Xu L, Hu Y, Cao Y, Li J, Ma L, Li Y, et al. An expression atlas of miRNAs in Arabidopsis thaliana. Sci China Life Sci. 2018;61: $178-89$.

69. Lister R, O'Malley RC, Tonti-Filippini J, Gregory BD, Berry CC, Millar AH, et al. Highly integrated single-base resolution maps of the epigenome in Arabidopsis. Cell. 2008;133:523-36.

70. He S, Vickers M, Zhang J, Feng X. Natural depletion of histone $\mathrm{H} 1$ in sex cells causes DNA demethylation, heterochromatin decondensation and transposon activation. eLife. 2019. https://doi.org/10.7554/elife.42530.

71. Boisnard-Lorig C, Colon-Carmona A, Bauch M, Hodge S, Doerner P, Bancharel E, et al. Dynamic analyses of the expression of the HISTONE::YFP fusion protein in arabidopsis show that syncytial endosperm is divided in mitotic domains. Plant Cell. 2001;13:495-509.

72. Teixeira FK, Heredia F, Sarazin A, Roudier F, Boccara M, Ciaudo C, et al. A role for RNAi in the selective correction of DNA methylation defects. Science. 2009:1600-4. https://doi.org/10.1126/science.1165313.

73. Kao P, Nodine MD. Transcriptional activation of Arabidopsis zygotes is required for initial cell divisions. Sci Rep. 2019;9: 17159.

74. Bouvet P, Dimitrov S, Wolffe AP. Specific regulation of Xenopus chromosomal 5 S rRNA gene transcription in vivo by histone H1. Genes Dev. 1994:8:1147-59.

75. Wolffe AP. Dominant and specific repression of Xenopus oocyte 5S RNA genes and satellite I DNA by histone H1. EMBO J. 1989;8:527-37

76. Mérai Z, Chumak N, García-Aguilar M, Hsieh T-F, Nishimura T, Schoft VK, et al. The AAA-ATPase molecular chaperone Cdc48/p97 disassembles sumoylated centromeres, decondenses heterochromatin, and activates ribosomal RNA genes. Proc Natl Acad Sci U S A. 2014;111:16166-71.

77. Jenik PD, Jurkuta REJ, Barton MK. Interactions between the cell cycle and embryonic patterning in Arabidopsis uncovered by a mutation in DNA polymerase epsilon. Plant Cell. 2005;17:3362-77.

78. Sonneville R, Craig G, Labib K, Gartner A, Blow JJ. Both chromosome decondensation and condensation are dependent on DNA replication in C. elegans embryos. Cell Rep. 2015;12:405-17.

79. Müller S, Almouzni G. Chromatin dynamics during the cell cycle at centromeres. Nat Rev Genet. 2017;18:192-208.

80. van Zanten M, Koini MA, Geyer R, Liu Y, Brambilla V, Bartels D, et al. Seed maturation in Arabidopsis thaliana is characterized by nuclear size reduction and increased chromatin condensation. Proc Natl Acad Sci U S A. 2011;108: 20219-24.

81. Pecinka A, Dinh HQ, Baubec T, Rosa M, Lettner N, Mittelsten SO. Epigenetic regulation of repetitive elements is attenuated by prolonged heat stress in Arabidopsis. Plant Cell. 2010;22:3118-29.

82. Probst AV, Mittelsten SO. Stress-induced structural changes in plant chromatin. Curr Opin Plant Biol. 2015;27:8-16.

83. Ito H, Gaubert H, Bucher E, Mirouze M, Vaillant I, Paszkowski J. An siRNA pathway prevents transgenerational retrotransposition in plants subjected to stress. Nature. 2011;472:115-9.

84. Masuta Y, Nozawa K, Takagi H, Yaegashi H, Tanaka K, Ito T, et al. Inducible transposition of a heat-activated retrotransposon in tissue culture. Plant Cell Physiol. 2017;58:375-84.

85. Zhang W, Wu Y, Schnable JC, Zeng Z, Freeling M, Crawford GE, et al. High-resolution mapping of open chromatin in the rice genome. Genome Res. 2012;22:151-62.

86. Lu X, Wontakal SN, Kavi H, Kim BJ, Guzzardo PM, Emelyanov AV, et al. Drosophila H1 regulates the genetic activity of heterochromatin by recruitment of Su(var)3-9. Sci Am Assoc Adv Sci. 2013;340:78-81.

87. Bošković A, Eid A, Pontabry J, Ishiuchi T, Spiegelhalter C, Raghu Ram EVS, et al. Higher chromatin mobility supports totipotency and precedes pluripotency in vivo. Genes Dev. 2014;28:1042-7.

88. Becker JS, Nicetto D, Zaret KS. H3K9me3-dependent heterochromatin: barrier to cell fate changes. Trends Genet 2016;32:29-41.

89. She W, Grimanelli D, Rutowicz K, Whitehead MWJ, Puzio M, Kotliński M, et al. Chromatin reprogramming during the somatic-to-reproductive cell fate transition in plants. Development. 2013:4008-19. https://doi.org/10.1242/dev.095034.

90. Rosa S, Ntoukakis V, Ohmido N, Pendle A, Abranches R, Shaw P. Cell differentiation and development in Arabidopsis are associated with changes in histone dynamics at the single-cell level. Plant Cell. 2014;26:4821-33.

91. Ikeuchi M, Favero DS, Sakamoto Y, Iwase A, Coleman D, Rymen B, et al. Molecular mechanisms of plant regeneration. Ann Rev Plant Biol. 2019:377-406. https://doi.org/10.1146/annurev-arplant-050718-100434.

92. Li H, Soriano M, Cordewener J, Muiño JM, Riksen T, Fukuoka H, et al. The histone deacetylase inhibitor trichostatin a promotes totipotency in the male gametophyte. Plant Cell. 2014;26:195-209.

93. Ikeda-Iwai M, Umehara M, Satoh S, Kamada H. Stress-induced somatic embryogenesis in vegetative tissues of Arabidopsis thaliana. Plant J. 2003;34:107-14.

94. Kamada H, Tachikawa Y, Saitou T, Harada H. Heat stress induction of carrot somatic embryogenesis. Plant tissue culture letters. Japanese Soc Plant Cell Mol Biol. 1994;11:229-32.

95. Li W, Liu H, Cheng ZJ, Su YH, Han HN, Zhang Y, et al. DNA methylation and histone modifications regulate de novo shoot regeneration in Arabidopsis by modulating WUSCHEL expression and auxin signaling. PLoS Genet. 2011;7:e1002243.

96. Henderson IR, Zhang X, Lu C, Johnson L, Meyers BC, Green PJ, et al. Dissecting Arabidopsis thaliana DICER function in small RNA processing, gene silencing and DNA methylation patterning. Nat Genet. 2006;38:721-5.

97. Onodera Y, Haag JR, Ream T, Costa Nunes P, Pontes O, Pikaard CS. Plant nuclear RNA polymerase IV mediates siRNA and DNA methylation-dependent heterochromatin formation. Cell. 2005;120:613-22.

98. Yelagandula R, Stroud H, Holec S, Zhou K, Feng S, Zhong X, et al. The histone variant H2A.W defines heterochromatin and promotes chromatin condensation in Arabidopsis. Cell. 2014;158:98-109.

99. Lutzmayer S, Enugutti B, Nodine MD. Novel small RNA spike-in oligonucleotides enable absolute normalization of small RNA-Seq data. Sci Rep. 2017:7:5913.

100. Martin M. Cutadapt removes adapter sequences from high-throughput sequencing reads. EMBnet.journal. 2011;17:10-2.

101. Lamesch P, Berardini TZ, Li D, Swarbreck D, Wilks C, Sasidharan R, et al. The Arabidopsis Information Resource (TAIR): improved gene annotation and new tools. Nucleic Acids Res. 2012;40:D1202-10. 
102. Dobin A, Davis CA, Schlesinger F, Drenkow J, Zaleski C, Jha S, et al. STAR: ultrafast universal RNA-seq aligner. Bioinformatics. 2013;29:15-21.

103. Schon MA, Kellner MJ, Plotnikova A, Hofmann F, Nodine MD. NanoPARE: parallel analysis of RNA $5^{\prime}$ ends from low-input RNA. Genome Research. 2018;28:1931-42.

104. Quinlan AR, Hall IM. BEDTools: a flexible suite of utilities for comparing genomic features. Bioinformatics. 2010;26:841-2.

105. Ramírez F, Ryan DP, Grüning B, Bhardwaj V, Kilpert F, Richter AS, et al. deepTools2: a next generation web server for deep-sequencing data analysis. Nucleic Acids Res. 2016;44:W160-5.

106. Krueger F, Andrews SR. Bismark: a flexible aligner and methylation caller for Bisulfite-Seq applications. Bioinformatics. 2011;27:1571-2.

107. Schultz MD, He Y, Whitaker JW, Hariharan M, Mukamel EA, Leung D, et al. Human body epigenome maps reveal noncanonical DNA methylation variation. Nature. 2015;523:212-6.

108. Stempor P, Ahringer J. SeqPlots - interactive software for exploratory data analyses, pattern discovery and visualization in genomics. Wellcome Open Res. 2016;1:14.

109. Chen F, Tillberg PW, Boyden ES. Expansion microscopy. Science. 2015:543-8. https://doi.org/10.1126/science.1260088.

110. Papareddy R, Nodine M. Chromatin regulates expression of small RNAs to help maintain transposon methylome homeostasis in Arabidopsis. GSE152971. Gene Expression Omnibus. https://www.ncbi.nlm.nih.gov/geo/query/acc. cgi?acc=GSE152971. Accessed 25 June 2020.

111. Papareddy R, Nodine M. Chromatin regulates expression of small RNAs to help maintain transposon methylome homeostasis in Arabidopsis. Github. https://github.com/mnodine/Papareddy.2020. Accessed 26 June 2020.

\section{Publisher's Note}

Springer Nature remains neutral with regard to jurisdictional claims in published maps and institutional affiliations.

Ready to submit your research? Choose BMC and benefit from:

- fast, convenient online submission

- thorough peer review by experienced researchers in your field

- rapid publication on acceptance

- support for research data, including large and complex data types

- gold Open Access which fosters wider collaboration and increased citations

- maximum visibility for your research: over $100 \mathrm{M}$ website views per year

At $\mathrm{BMC}$, research is always in progress.

Learn more biomedcentral.com/submissions 